\title{
APPROXIMATION OF CERTAIN FUNCTIONS BY EXPONENTIALS ON A HALF LINE
}

\author{
PAUL KOOSIS
}

Introduction. In a recent paper, [1], A. Beurling has shown that the positive translates of an integrable function defined on $[0, \infty)$ generate, in a certain sense, at least one exponential of the form $e^{-i a x}, x \geq 0, I a<0$, provided that the function does not vanish outside a finite interval. It is the converse problem with which we shall be concerned here; namely, to what extent can the exponentials so generated be used to approximate the given function. We are able to give what amounts to a complete solution.

The situation resembles strongly that of Schwartz' theory of mean periodic functions [2]. M. Kahane has shown in [3] (see also [4]) how this theory can be presented very simply using the notion of Fourier transform of a mean periodic function. Beurling also made use of this method in the present case; however, we shall find it convenient to exploit this tool more systematically, in closer analogy with Kahane's work. We shall also study our approximations in a topology (the same as the one used in the theory of mean periodic functions) which is simpler than that of Beurling.

Beurling based his work mainly on a certain division theorem which states roughly that an entire function is of finite exponential type if it is bounded on a half plane and equal to the ratio of two bounded analytic functions on the complementary half plane. The conclusions we make here follow from a refinement of this given in $\$ 3$ which yields an upper bound for the type of such an entire function.

It should be remarked that B. Nyman ([7, pp. 28-29]) has established a result similar to the one given here, using, however, a quite different topology. (The referee calls attention to this in his report; although I have since had the opportunity to consult Nyman's work, it was not accessible to me in New York at the first writing of this paper.)

1. We shall consider functions $f \in L_{1}(-\infty, \infty)$ which are continuous on $[0, \infty)$ and vanish on $(-\infty, 0)$. Together with such functions $f$ we also consider their positive translates $f_{h}, f_{h}(x)=f(x+h), h \geq 0$. $E$ denotes the space of continuous functions on $[0, \infty)$, and $E_{f}$ that subspace of $E$ consisting of functions which can be uniformly ap-

Received by the editors April 2, 1956 and, in revised form, July 7, 1956. 
proximated on compacta by linear combinations of the $f_{h}$ (restricted to $[0, \infty)), h \geq 0$.

1.1. Definition. $f$ is said to be mean periodic on $[0, \infty)$ if and only if $E_{f}$ is a proper subset of $E$.

A standard argument due to $\mathrm{F}$. Riesz ${ }^{1}$ shows that $f$ is mean periodic on $[0, \infty)$ if and only if there exists a nonzero measure $\tilde{m}$ of compact support in $[0, \infty)$ so that $\int_{0}^{\infty} f_{h}(x) d \tilde{m} x=0, h \geq 0$. If $m$ is any measure, we use systematically the notation:

$$
d \tilde{m} x=d m(-x), \quad \tilde{m}^{*}=m .
$$

Then, the above condition that $f$ be mean periodic on $[0, \infty)$ is that for some $m \neq 0$ of compact support in $(-\infty, 0], m * f=0$ on $[0, \infty)$. (As is customary, we write $(m * f)(x)=\int_{-\infty}^{\infty} f(x-y) d m y ; m * f$ is the convolution of $m$ with $f$.)

1.2. Definition, For $f$ mean periodic on $[0, \infty), L_{f}$ denotes the infimum of all $L>0$ so that there exists a measure $m \neq 0$ having support in $[-L, 0]$ with $m * f=0$ on $[0, \infty)$.

Evidently, if $L_{f}>0$ and $0<k<L_{f}$, any continuous function on $[0, k]$ can be uniformly approximated by linear combinations of the $f_{h}$, $h \geq 0$, while for $k>L_{f}$ this is no longer true. For $m \neq 0$ of compact support in $(-\infty, 0]$ such that $m * f=0$ on $[0, \infty)$ let $g=m * f$. Then $g$ is also of compact support in $(-\infty, 0]$, vanishing outside the smallest interval containing the support of $m$. We indicate the Fourier transform of any measure or function by placing a circumflex over the symbol denoting it, e.g.

$$
\hat{m}(\lambda)=\int_{-\infty}^{\infty} e^{i \lambda x} d m x .
$$

$\hat{g}(\lambda)$ and $\widehat{m}(\lambda)$ are then entire functions of finite exponential type, so

$$
\hat{f}(\lambda)=\frac{\hat{g}(\lambda)}{\hat{m}(\lambda)}
$$

possesses a meromorphic extension to the entire complex plane. Since

${ }^{1}$ Let $F$ be any subspace of $E$ closed with respect to uniform convergence on compacta. If $g$ is a function in $E \sim F$, then there exists a measure $m$ of compact support in $[0, \infty)$ so that $\int g d m \neq 0$ whilst $\int u d m=0$ for $u \in F$. (The converse of this is evident.) For there must be a $\delta>0$ and a finite interval $J$ in $[0, \infty)$ such that for no $u \in F$ is $|g-u|<\delta$ on $J$. Let $B$ denote the Banach space of continuous functions on $J$ under the uniform norm, and $F^{\prime}$ the closed subspace of $B$ generated by the restrictions of the members of $F$ to $J$. If $g^{\prime}$ is the restriction of $g$ to $J, g^{\prime} \in B \sim F^{\prime}$. By the Hahn-Banach theorem, there must be a continuous functional $A$ on $B$ so that $A g^{\prime} \neq 0, A u^{\prime}=0$ for $u^{\prime} \in F^{\prime}$. By the Riesz representation theorem there is then a measure $m$ with support in $J$ so that $A v=\int v d m, v \in B$. This leads immediately to the desired conclusion. 
$f=0$ on $(-\infty, 0)$ and $f \in L_{1}, \hat{f}(\lambda)$ is regular and bounded for $I \lambda \geq 0$.

1.3. Theorem. A necessary and sufficient condition that the function $x^{n} e^{-i a x}(x \geq 0)$ be in $E_{f}$ is that a be at least an $n+1$ fold pole of $\hat{f}(\lambda)$.

The proof is the same as that of Theorem 1 in [3], but for the reader's convenience, it is reproduced here.

Consider the case $n=0$. Suppose $a$ is a pole of $\hat{f}(\lambda)$. By the above formula for $\hat{f}(\lambda)$, we must have $\hat{m}(a)=0$ for every measure $m$ of compact support in $(-\infty, 0]$ such that $m * f=0$ on $[0, \infty)$. On restating this in terms of $\tilde{m}$ and using the result given in the footnote, we see that $e^{-i a x} \in E_{f}$.

If, conversely, $e^{-i a x} \in E_{f}$, pick a nonzero $m$ of compact support in $(-\infty, 0]$ so that $m * f$ vanishes on $[0, \infty)$. We must then have $\hat{m}(a)$ $=0$, and we are done unless also $\hat{g}(a)=0$. In that case, consider the functions $M=m * K, G=g * K$, where

$$
K(x)= \begin{cases}e^{-i a x}, & x \geq 0, \\ 0, & x<0 .\end{cases}
$$

(The convolution of a function $g$ with another function is defined the same way as that of a measure $m$ with another function was defined above, save that we use $g(x) d x$ instead of $d m x$.)

Since $m$ and $g$ have compact supports in $(-\infty, 0]$ and $\hat{m}(a)=\hat{g}(a)$ $=0$, we see that $M$ and $G$ have compact supports in $(-\infty, 0)$, and it is legitimate (remembering that $f=0$ on $(-\infty, 0)$ ) to apply the usual commutivity and associativity rules for convolutions to conclude $G=(m * f) * K=(m * K) * f=M * f$. In particular, $M * f=G$ vanishes on $[0, \infty)$, whence $\hat{M}(a)=0$, using again the fact that $e^{-i a x} \in E_{f}$. Now

$$
\hat{K}(\lambda)=\frac{i}{\lambda-a},
$$

$I \lambda>I a$,

whence by analytic continuation $\hat{G}(\lambda)=i \hat{g}(\lambda) /(\lambda-a)$ for all $\lambda$. Since $\hat{f}(\lambda)=\hat{G}(\lambda) / \hat{M}(\lambda)$, we are done if the zero of $\hat{g}(\lambda)$ at $a$ is of order one. Otherwise, the argument may be repeated to attain the desired conclusion.

In case $n>0$, one notes that if $x^{n} e^{-i a x} \in E_{f}$, then $x^{m} e^{-i a x} \in E_{f}$ for $m=0,1, \cdots, n-1$, this following immediately from the definition of $E_{f}$. The theorem for the general case is then established in the same way as above.

From the above theorem and the fact that $\hat{f}(\lambda)$ is regular and bounded in $I \lambda \geq 0$, it follows that we can have $x^{n} e^{-i a x} \in E_{f}$ only for Ia $<0$. 
1.4. Definition. $X_{f}$ denotes the set of functions ("exponential monomials") of the form $x^{n} e^{-i a x}$ in $E_{f}$.

The question with which we are concerned in this paper is whether or not a given $f$ mean periodic on $[0, \infty)$ can be uniformly approximated on compact subsets of $[0, \infty)$ by linear combinations of members of $X_{f}$. It turns out that this is not true, but that $f_{h}$ can be so approximated for $h$ sufficiently large.

1.5. Definition. $A_{f}$ denotes the infimum of all $h \geq 0$ such that $f_{h}$ can be approximated in the way mentioned above.

Our problem reduces to the study of the relation between the numbers $A_{f}$ and $L_{f}$.

We need one last preliminary result:

1.6. Theorem. Let $f$ be mean periodic on $[0, \infty)$ and let $m \neq 0$ be a measure of compact support in $(-\infty, 0]$ so that $m * f=0$ on $[0, \infty)$. Let $p$ be any measure of compact support in $(-\infty, 0]$ and let

$$
u(x)= \begin{cases}(p * f)(x), & x \geq 0, \\ 0, & x<0 .\end{cases}
$$

Then $u$ is mean periodic on $[0, \infty)$ and $m * u=0$ there. Moreover, the set of poles of $a(\lambda)$ is the set of poles of $\hat{f}(\lambda)$ diminished by the set of zeros of $\hat{p}(\lambda)$ (taking into account multiplicities).

Proof. The first statement follows because $f$ vanishes off $[0, \infty)$ while $\tilde{m}$ and $\tilde{p}$ have their supports there. Again because of this, $u=p * f+$ a function of compact support, so $\hat{u}(\lambda)=\widehat{p}(\lambda) \hat{f}(\lambda)+$ an entire function, and the second statement follows.

2. We shall prove our division theorem.

2.1. Lemma. Let $H(z)=\int_{0}^{1} e^{-i z t} d m t, m \neq 0$, and let, for $y \geq 0, M(y)$ $=\sup _{x}|H(x+i y)|$. Then always $M(y)>0$ and for any $\epsilon>0$ there is an integer $k>0$ so that $M(k) / M(k-1) \leq e^{1+\epsilon}$.

Proof. That $M(y)>0$ follows from $m \neq 0$ by a standard uniqueness theorem. If the second conclusion is false, then for some $\epsilon>0, M(k)$ $\geqq M(0) e^{(1+\epsilon) k}$ for $k$ integers $\rightarrow \infty$, whence $\lim \sup _{y \rightarrow \infty} \log M(y) / y$ $\geqq 1+\epsilon$, which contradicts the definition of $H(z)$.

2.2. Theorem. Let $F(z)=G(z) / H(z)$ be analytic everywhere, with $G(z)$ and $H(z)$ entire. Suppose

1. $|F(z)|$ is bounded for $I z \geq 0$.

2. For $k>0,|G(z)|$ is bounded for $I z \leq k$.

3. $H(z)=\int_{0}^{A} e^{-i z t} d m t, m \neq 0$.

Then, for any $\epsilon>0$ there exists a constant $K$ so that 


$$
|F(z)| \leq K e^{(\Lambda+\epsilon)|z|}
$$

Proof. By a change of variable, we may reduce the theorem to the case $A=1$, which we suppose done. Retaining the notation of 2.1 , choose an integer $k>0$ so that $\log (M(k) / M(k-1)) \leq 1+\epsilon / 4$, which is possible by that lemma. By definition of $M(y)$ we can then find a real $c$ so that

$$
\ln \frac{M(k)}{|H(c+(k-1) i)|} \leq 1+\frac{\epsilon}{2} .
$$

By means of the transformation

$$
z \rightarrow w=\frac{i+(z-c-i k)}{i-(z-c-i k)}
$$

map $I z \leq k$ conformally onto $|w| \leq 1$ so that $c+(k-1) i$ goes into 0 . $|H(z)|$ is bounded for $I z \leq k$, hence by the Phragmén-Lindelöf theorem, $|H(z)| \leq M(k)$ for $I z \leq k$. Put $H(z) / M(k)=h(w), G(z)=g(w)$ for $z \rightarrow w$ in the above mapping. We have, for $|w|<1,|h(w)| \leq 1$ and by hypothesis $|g(w)| \leq$ some number $C$.

By a well-known theorem $\left[5\right.$, p. 188] we can write $g(w)=P_{1}(w) g_{1}(w)$, $h(w)=P_{2}(w) f(w)$, where $P_{1}(w)$ and $P_{2}(w)$ are Blaschke products, $g_{1}(w)$ and $f(w)$ are free of zeros in $|w|<1$; moreover, $\left|P_{1}(w)\right| \leq 1$, $\left|P_{2}(w)\right| \leq 1,\left|g_{1}(w)\right| \leq C$, and $|f(w)| \leq 1$ there. Since $g(w) / M(k) h(w)$ $=F(z)$ is free of poles in $|w|<1$, some of the zeros of $P_{1}(w)$ must cancel those of $P_{2}(w)$, and we have $P_{1}(w) / P_{2}(w)=P(w)$, another Blaschke product, $|P(w)| \leq 1$ in $|w|<1$. We therefore have, for

$$
I z<k, \quad|F(z)| \leq \frac{C}{M(k)|f(w)|} .
$$

Since $|f(w)| \leq 1$ and $f(w)$ is free of zeros in $|w|<1$, we can write [5, p. 197]:

$$
\ln \left|f\left(r e^{i \theta}\right)\right|=-\frac{1}{2 \pi} \int_{0}^{2 \pi} \frac{\left(1-r^{2}\right) d \mu \phi}{1+r^{2}-2 r \cos (\theta-\phi)}
$$

for $\mu$ a positive measure on $[0,2 \pi]$. Therefore

$$
\log |h(0)|=\log \left|P_{2}(0)\right|+\log |f(0)| \leq-\frac{1}{2 \pi} \int_{0}^{2 \pi} d \mu \phi
$$

and

$$
\frac{1}{2 \pi} \int_{0}^{2 \pi} d \mu \phi \leq-\log |h(0)|=\log \left|\frac{M(k)}{H(c+(k-1) i)}\right| \leqq 1+\frac{\epsilon}{2}
$$


by choice of $k$ and $c$. This, combined with (3) yields

$$
-\log |f(w)| \leq \frac{4}{1-|w|^{2}}\left(1+\frac{\epsilon}{2}\right) .
$$

For $z=R e^{i \Phi}, \pi \leq \Phi \leq 2 \pi$, we have, on substituting in (1),

$$
\frac{4}{1-|w|^{2}}=\frac{(1+k)^{2}+c^{2}-2 R((1+k) \sin \Phi+c \cos \Phi)+R^{2}}{k-R \sin \Phi} .
$$

Since $k>0$, this expression is

(i) $\leq$ const $+R / \sin b, \pi+b \leq \Phi \leq 2 \pi-b, b$ any fixed number between 0 and $\pi / 2$;

(ii) $\leq$ const $+B^{\prime} R^{2}$ for some $B^{\prime}, \pi \leq \Phi \leq 2 \pi$.

Combining this with (2) and (4) we find:

$$
\begin{array}{lr}
\left|F\left(R e^{i \Phi}\right)\right| \leq \text { const } \cdot \exp \frac{(1+\epsilon / 2) R}{\sin b}, & \pi+b \leq \Phi \leq 2 \pi-b, \\
\left|F\left(R e^{i \Phi}\right)\right| \leq \text { const } \cdot \exp B R^{2}, & \pi \leq \Phi \leq 2 \pi .
\end{array}
$$

Now $|F(z)|$ is by hypothesis bounded on the real axis, so, applying the Phragmén-Lindelöf theorem in each of the sectors $\pi \leq \Phi \leq \pi+b$, $2 \pi-b \leq \Phi \leq 2 \pi$, we find

$$
|F(z)| \leq \text { const } \cdot \exp \frac{(1+\epsilon / 2)|z|}{\sin b}
$$

holds in the lower half plane. Making $b$ close enough to $\pi / 2$, we get $|F(z)| \leq K e^{(1+e)|z|}$, which holds also in the upper half plane, since by hypothesis $|F(z)|$ is bounded there. Q.E.D.

3. We are now able to establish the main result:

3.1. Theorem. For $f$ mean periodic on $[0, \infty), 0 \leq A_{f} \leq L_{f}$.

PROof. In order to show that $f_{h}$ is uniformly approximable by linear combinations of members of $X_{f}$ on compact subsets of $[0, \infty)$, it is enough to show that for any measure $\tilde{p}$ of compact support therein which satisfies

$$
\int_{0}^{\infty} x^{n} e^{-i a x} d \tilde{p} x=0 \text { for all functious } x^{n} e^{-i a x} \text { in } X_{f},
$$

one has $\int_{0}^{\infty} f_{h}(x) d \tilde{p} x=0$. (See above footnote.) Supposing that $\tilde{p}$ is such a measure, define

$$
u(x)= \begin{cases}(p * f)(x), & x \geq 0, \\ 0, & x<0 .\end{cases}
$$


We will be done if we show that $u(x)$ vanishes for $x>L_{f}$.

Let $\tilde{m} \neq 0$ be a measure on $\left[0, L_{f}+\epsilon\right]$ so that $m * f=0$ on $[0, \infty)$. By 1.6, $m * u=v$ has compact support in $(-\infty, 0]$ and our assumption on $\tilde{p}$ means by 1.3 that $\hat{p}(\lambda)$ vanishes at all the poles of $\hat{f}(\lambda)$, so by 1.6 again, $\hat{u}(\lambda)$ is analytic everywhere. $a(\lambda)=\widehat{v}(\lambda) / \widehat{m}(\lambda) ; \widehat{v}(\lambda)$ is obviously bounded for $I \lambda \leq k, k>0$, and

$$
\widehat{m}(\lambda)=\int_{0}^{L_{f}+\epsilon} e^{-i \lambda x} d \tilde{m} x,
$$

while $\hat{a}(\lambda)$ is bounded for $I \lambda \geq 0$ as in the remarks following 1.2. We may therefore apply 2.2 to conclude $|a(\lambda)| \leq K \exp \left(L_{f}+2 \epsilon\right)|\lambda|$.

By an obvious modification of the Paley-Wiener theorem [6, p. 109], it follows that $u(x)=0, x \geq L_{f}+2 \epsilon$, and squeezing $\epsilon$, we obtain the required result.

3.2. Corollary. If $f$ is mean periodic on $[0, \infty)$ and $X_{f}=\{0\}$, then $f(x)=0$ for $x>L_{f}$.

3.3. We show finally by means of two examples that the inequality $0 \leq A_{f} \leq L_{f}$ established in 3.1 cannot be improved.

EXAMPLE 1. Let

$$
f(x)= \begin{cases}\sum_{-\infty}^{\infty} a_{n} e^{-x} e^{i n x}, & x \geq 0, \\ 0, & x<0,\end{cases}
$$

where the $a_{n}$ are chosen all $>0$ so that their infinite sum converges. It is easy to see (e.g., on computing $\hat{f}(\lambda)$ and using 1.3) that $X_{f}$ $=\left\{e^{-x} e^{i n x}\right\}$ whence $A_{f}=0 . X_{f}$ is clearly uniformly complete on any interval $[0, k], k<2 \pi$, since for a given continuous $q$ on such an interval we may approximate $e^{x} q(x)$ uniformly by linear combinations of the $e^{i n x}$ thereon. But for $k \geq 2 \pi$, this is no longer true; only those continuous functions $q$ for which $q(x+2 \pi)=e^{-2 \pi} q(x)$ can be so approximated.

So here $L_{f}=2 \pi$, whereas $A_{f}=0$.

EXAMPLE 2. Let

$$
f(x)=\left\{\begin{array}{lr}
e^{-x}, & x \geq 1, \\
e^{-1}, & 0 \leq x \leq 1, \\
0, & x<0 .
\end{array}\right.
$$

For any $\epsilon>0$ we can clearly find a measure $\tilde{m}$ on $[1,1+\epsilon]$ so that $\int_{1}^{1+\epsilon} e^{-x} d \tilde{m} x=0$. If we extend $\tilde{m}$ so as to be zero on $[0,1)$ we will have $\int_{0}^{1+e} f_{h}(x) d \tilde{m} x=0, h \geq 0$. Therefore $L_{f} \leq 1$. Clearly, $X_{f}=\left\{e^{-x}\right\}$ and since 
$f(x)=$ const on $[0,1], A_{f}=1$. So here $A_{f} \geq L_{f}$; i.e., in conjunction with $3.1, A_{f}=L_{f}$. (It is also easy to show directly that $L_{f}=1$.) In conclusion, I would like to express my thanks to Dr. Peter Lax, who introduced me to this subject, and with whom I have had many helpful discussions.

\section{REFERENCES}

1. A. Beurling, $A$ theorem on functions defined on a semigroup, Math. Scand vol. 1 (1953) pp. 127-130.

2. L. Schwartz, Théorie générale des fonctions moyenne-périodiques, Ann. of Math. vol. 48 (1947) pp. 857-929.

3. J.-P. Kahane, Sur quelques problèmes d'unicité et de prolongement . . ., Annales de l'Institut Fourier vol. 5 (1953-1954) Ch. I, §1, pp. 41-48.

4. P. Koosis, Note sur les fonctions moyenne-périodiques, Annales de l'Institut Fourier vol. 6 (1955-1956) pp. 357-360.

5. R. Nevanlinna, Eindeutige analytische Funktionen, Berlin-Springer, 2d ed., 1953.

6. R. Boas, Entire functions, New York, Academic Press, 1954.

7. B. Nyman, On the one-dimensional translation group and semi-group in certain function spaces, thesis, Uppsala, 1950.

New YoRk UNIVERSITY

\section{FUNCTIONAL EQUATIONS IN THE THEORY OF DYNAMIC PROGRAMMING-VII. A PARTIAL DIFFERENTIAL EQUATION FOR THE FREDHOLM RESOLVENT}

\section{RICHARD BELLMAN}

1. Introduction. Let $K(x, y)$ be a symmetric kernel over the square $0 \leqq x, y \leqq T$, continuous in both variables in this region, and possessing the additional property that $\int_{0}^{T} \int_{0}^{T} K(x, y) u(x) u(y) d x d y+\int_{0}^{T} u^{2}(x) d x$ is positive definite. Then the Fredholm integral equation

$$
u(x)+v(x)+\int_{a}^{T} K(x, y) u(y) d y=0, \quad 0 \leqq a \leqq T,
$$

has a unique solution for any function $v(x)$ continuous for $a \leqq x \leqq T$. This solution may be represented in the form

$$
u(x)=-v(x)+\int_{a}^{T} Q(x, y, a) v(y) d y .
$$

Let us call the kernel $Q(x, y, a)$ the Fredholm resolvent.

The purpose of this note is to show that $Q(x, y, a)$ satisfies the

Received by the editors August 2, 1956 and, in revised form, September 24, 1956. 